OPEN ACCESS

Edited by:

Agostino Mattei,

Luzerner Kantonsspital, Switzerland

Reviewed by:

Mohammad Abufaraj, Medizinische Universität Wien, Austria

Francesco Soria,

Universität Wien, Austria

Giovanni Battista Di Pierro,

Università degli Studi di Roma La

Sapienza, Italy

*Correspondence:

Fabrizio Dal Moro

fabrizio.dalmoro@gmail.com

Specialty section:

This article was submitted to

Genitourinary Surgery,

a section of the journal

Frontiers in Surgery

Received: 21 July 2018 Accepted: 17 September 2018

Published: 05 October 2018

Citation:

Cattaneo F, Motterle G, Zattoni F,

Morlacco A and Dal Moro F (2018)

The Role of Lymph Node Dissection in

the Treatment of Bladder Cancer.

Front. Surg. 5:62

doi: 10.3389/fsurg.2018.00062

\section{The Role of Lymph Node Dissection in the Treatment of Bladder Cancer}

\author{
Francesco Cattaneo ${ }^{1}$, Giovanni Motterle ${ }^{1}$, Filiberto Zattoni ${ }^{1}$, Alessandro Morlacco ${ }^{1}$ and \\ Fabrizio Dal Moro ${ }^{1,2 *}$
}

${ }^{1}$ Clinica Urologica, Dipartimento di Scienze Chirurgiche, Oncologiche e Gastroenterologiche, Università degli Studi di Padova, Padova, Italy, ${ }^{2}$ Clínica Urologica, Ospedale "Santa Maria della Misericordia", Università di Udine, Udine, Italy

Lymph node dissection (LND; PLND: pelvic LND) is an essential component of radical cystectomy $(\mathrm{RC})$ for bladder cancer $(\mathrm{BC})$. However, the optimal anatomical extent of LND and its potential therapeutic role are still controversial: as we will explain, the extent of LND dissection is a predictor of survival and local recurrence but what is an adequate extension is still unclear. Moreover, there is large uncertainty about the role of surgery in patients with clinically-positive nodes. In this review we will provide a synthesis of the available evidence on this highly debated topic. Overall, the studies presented in this work support the idea that extended lymphadenectomy could provide optimal diagnostic and possibly therapeutic results in cN- patients. In cN+ patients, post chemotherapy surgery may be considered especially in subjects who have a good response to $\mathrm{CHT}$, although definitive evidence is still needed. Finally, the final results of randomized trials are eagerly awaited to draw definitive conclusions of the role of PLND in BC.

Keywords: bladder cancer, lymph nodes, lymph node dissection, radical cystectomy, nodal disease

\section{INTRODUCTION}

Radical cystectomy (RC) plus regional lymph node dissection (LND) is the gold standard in the treatment of high-risk non-muscle-invasive bladder cancer (NMIBC) unresponsive to intravesical therapies or muscle-invasive bladder cancer (MIBC). Neoadjuvant chemotherapy is recommended as part of the treatment (for cT2-T4a N0 MIBC. RC includes the removal of the bladder itself and its surrounding perivescical fat. In men RC consists in removing also the prostate and seminal vesicles whereas in woman it includes the ovaries, uterus with cervix, and anterior vagina (1).

LND is an essential step in the treatment of MIBC because it is known that approximately $25-30 \%$ of patients will have lymph node metastasis at the time of surgery $(2-4)$ and also because lymph node status is one of the most important indicators of long term overall survival (OS) and recurrence-free survival (RFS) (5).

As we will analyze more in detail in this paper, the extent of LND dissection is a predictor of survival and local free recurrence but what is an adequate extension is still unclear.

The rationale of removing any positive lymph node appears obvious because it provides a more complete removal of cancer and a better stratification and staging of the patient for further adjuvant therapies; however, the evidence supporting this approach is limited in quality, quantity, and somewhat controversial. According to the known pathophysiology of BC metastases, LND might be a helpful step also in $\mathrm{pN} 0$ patients because it could help remove immunosuppressive factors that could to facilitate distant metastases. 


\section{ANATOMICAL PERSPECTIVE OF LYMPH NODE DISSECTION FOR BLADDER CANCER}

The primary lymphatic drainage site for bladder cancer includes the internal iliac, external iliac, obturator, and presacral lymph nodes. Secondary drainage sites include the common iliac, paraaortic, interaortocaval, and paracaval lymph nodes (Figures 1, 2) $(6,7)$.

Although "skip" metastasis (meaning metastases in secondary drainage sites without evidence of metastases in primary sites) appears to be a relatively rare event in bladder cancer, it has been reported in the literature. Leissner et al. in their series found that $6.9 \%$ of patients had nodal metastasis in lymph nodes above the common iliac bifurcation but none above the aortic bifurcation; therefore, to achieve an accurate LN staging, it would be necessary to dissect up to the aortic bifurcation. (8).

Tarin et al. (9) evaluated 591 RC patients. LN involvement was identified in 114 patients (19\%). Stratifying by tumor stage $<$ pT2, pT2, pT3, and pT4, LN involvement was identified in 6, 18,40 , and 12 patients $60 \%$, respectively. In this group, seven patients $(6 \%)$ had no positive lymph nodes within the true pelvis (skip lesions). Since skip lesions are known to be very rare, this phenomenon may be the result of missed positive LNs in the true pelvis or of a specimen-labeling error.

Also mapping studies have found that a very low number of patients had metastases in regions above the bifurcation of aorta without synchronous metastases in the true pelvis. These observations strengthens the idea that "skip" metastasis is an exception rather than the rule (9).

In a recent series Moschini et al. evaluated 653 patients with $\mathrm{cN} 0 \mathrm{cM} 0$ high risk NMIBC or MIBC treated with RC and extended or super-extended PLND without neoadjuvant CHT. $29.3 \%$ of patients had pathologically confirmed node metastasis. Most patients were found with node metastases within the standard template $(26.3 \%)$, on the other hand $4.6 \%$ and $3.2 \%$ patients had node metastases in extended and super-extended templates, respectively. However, of these only 2 patients were

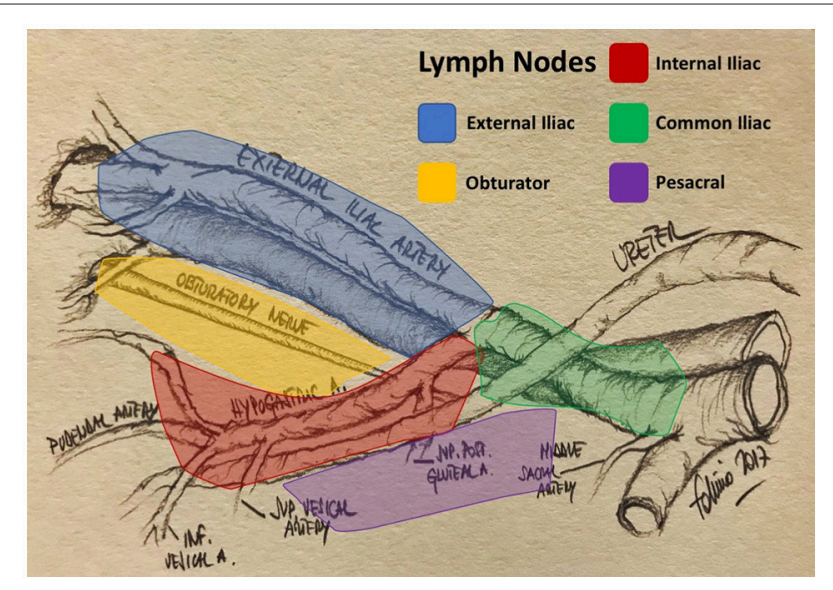

FIGURE 1 | Pelvic lymph node areas (original drawing by the author). found without concomitant lymph node metastases in the limited or standard templates (3). According to this study, superextended LND template might be superfluous in the large majority of patients for staging alone, since most patients with positive nodes in the extended or superextended templates will have positive nodes also in the limited or standard fields.

\section{PATTERNS OF NODAL SPREAD IN MUSCLE-INVASIVE BLADDER CANCER}

One autopsy study evaluated the metastatic behavior of bladder tumors and showed that, in 251 of 367 patients (68\%) with metastatic MIBC, the most frequent sites of metastases were: regional lymph nodes (90\%: $92 \%$ in perivesical or pelvic, $72 \%$ in retroperitoneal, and $35 \%$ in abdominal lymph nodes), liver (47\%), lung (45\%), bone (32\%), peritoneum (19\%), pleura (16\%), kidney (14\%), adrenal gland (14\%), and the intestine (13\%). The frequency of metastases increased with local tumor extension and

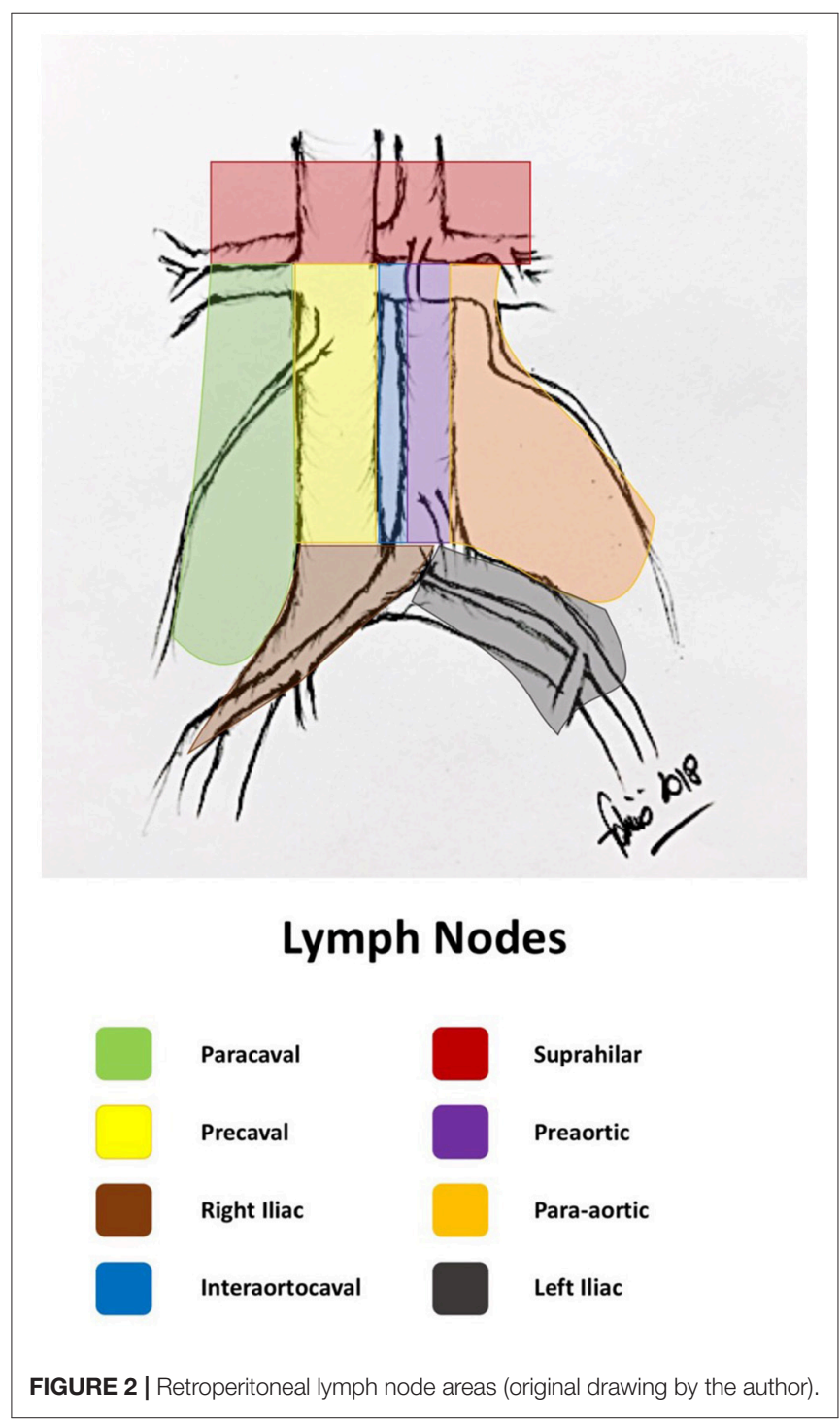


there was a strong association between the presence of lymph node and distant metastases (47\% of the patients had both). This association suggests that distant metastases could mostly be derived from the regional metastases and as a consequence lymphadenectomy could theoretically improve the prognosis (10).

LND plays an important role also in high risk NMIBC undergoing RC as we can see from Bruins' work where of 114 patients who underwent RC for NMIBC, $9 \%$ with cT1, $12 \%$ with cTis, and $0 \%$ with cTa had lymph node metastasis (4).

\section{MORE IS BETTER? EXTENT OF LND}

Proven the importance of LND as part of staging and treatment of BC, there are still some questions that need to be answered. Does the extent of LND give a survival benefit? And which is more important between the anatomic extent of dissection and the number of nodes removed?

The anatomic extent of PLND necessary for loco-regional disease control and reliable staging is controversial, and currently no dissection template has been universally accepted. Furthermore, there has been no uniformity in reporting and measuring the dissection extent; thus, the most commonly used measure of the extent of PLND has been the lymph node count.

However, although descriptions of the anatomic extent of lymphadenectomy somewhat differs among the published studies, the extent of LND was determined a priori based on discussion in an expert panel (EAU Working Group on MIBC) and was categorized as follows:

- Limited is defined as the removal of obturator and internal iliac nodes,

- Standard included also the external iliac nodes,

- Extended included also common and presacral nodes,

- Super-extended included all the nodes removed along the inferior mesenteric artery (11).

It has been shown that limited PLND removes only about $50 \%$ of all primary lymphatic landing sites. In order to remove $90 \%$, PLND should be extended to include LNs lateral and medial to the internal iliac vessels, and the common iliac region up to the uretero-iliac crossing (12).

Several factors might influence lymph node count such as the method of lymph node submission (en-bloc vs. separate packets and the number of packets sent), surgical technique, and variability in the pathologic practices and reporting standards. Last but not least, there is an important inter-individual variability in the number of lymph nodes that can be retrieved from the same template (13).

Reports in the literature regarding the correlation between the number of dissected nodes and the prognosis following RC are conflicting. Li et al. performed a meta-analysis of 41.400 patient who underwent $\mathrm{RC}$, of which 6.044 were $\mathrm{pN}+$. In their study they showed that a greater extent of LND during RC had statistically significant advantages in terms of OS, CSS and RFS, corresponding to reduced risks of 28,34 and $36 \%$, respectively, compared with patients with a lesser extent of LND (14).
The number of resected nodes showed positive correlation also regarding local recurrence rates $(p=0.002$ in $>11$ nodes in $\mathrm{pN}+$ patients) (15) and a stronger association with survival (HR 2.0, $p=0.001)$ (16).

Cole et al. used the SEER data to analyze adequacy of LND (defined by $>10$ nodes removed) during years from 1988 to 2010 and found that, in the total sample, only $45 \%$ of patients received and adequate LND, with a proportion increasing over time from 26.4 to $61.3 \%$ (17).

Dhar and al. found that 5-years RFS was 23 vs. $57 \%$ $(p<0.0001)$, and OS was 26 vs. $46 \%(p=0.0021)$, in favor of the extended LND group compared to limited LND group. For $\mathrm{pN}+$ patients the 5-year relapse-free survival and overall survival were both $7 \%$ for a limited dissection compared with 35 and 34\% for patients undergoing extended LND, respectively $(p<0.0001)$ (18).

A super-extended dissection (up to the inferior mesenteric artery) resulted in higher node count (median 38 vs. 22, $p<0.0001$ ) without survival benefit (19). This lack of survival advantage was confirmed in another study from Bruins (11).

Other retrospective series examined the relation between the extent of lymph node dissection, as defined by the number of lymph nodes removed, and survival in patients with or without lymph node metastases (20). Data supported the role of extended PLND in improving survival in both node positive and node negative $\mathrm{BCa}$ patients $(18,20)$. Considering demographics and pathological features, clinical $\mathrm{T}$ and $\mathrm{N}$ stage were predictors of the possibility to harbor node metastases in the extended or super-extended template (3).

In a meta-analysis of all studies comparing extended and standard PLND, overall odds ratio of 5-year recurrence-free survival rate was 1.63 (95\% CI 1.28-2.07, $p<0.001$ ), suggesting a significant benefit for the extended PLND with no increase in mortality and/or morbidity (21). In a recent systematic review, the influence of LND on perioperative and oncologic outcomes in patients undergoing RC for MIBC was assessed including 23 studies reporting on 19.793 patients (11). Of interest, in this last study, the meta-analysis originally planned by the authors was not possible, due to the large heterogeneity between studies. However, the final results suggested that in terms of oncologic outcomes, LND of any extent is better than no LND; furthermore, extended LND might improve oncologic outcomes compared with more limited types of dissection, although extending the dissection beyond the boundaries of eLND (i.e., super-extended LND) is unlikely to lead to any further benefits. Despite the evidence summarized in this review was not strong enough to provide firm recommendations regarding the most optimal extent of LND, the included studies fairly consistently report an oncologic benefit for eLND compared with less extended LND templates.

On the other hand, preliminary available prospective evidence did not prove a survival benefit of extended PLND compared to limited PLND. The phase 3 trial conducted of Association of Urogenital Oncology and German Cancer Association (LEA trial) examined recurrence free survival (primary endpoint) and cancer-specific survival (secondary endpoint) of extended PLND (including 14 fields, up to the inferior mesenteric artery) vs. limited PLND (including 6 fields: bilateral obturator, internal and 
external iliac nodes). There was only a trend but no significant difference in terms of improved RFS and CSS with an extended PLND. On a post-hoc analyses, a survival benefit was seen only for patients who harbored an organ confined disease (22).

Another randomized clinical trial of the National Cancer Institute (SWOG 1011), active in USA and Canada, is still in progress, with the primary aim of examining disease free survival of extended (including common iliac and presacral PLND) vs. standard PLND (23). The estimated end of this study is 2022 and the planned enrollment 620 participants.

Therefore, further data from on-going randomized clinical trials on the therapeutic impact of the different extents of lymphadenectomy are awaited.

The diagnostic performances and the survival outcomes of the articles presented in the text are summarized in Table $\mathbf{1}$.

\section{TREATMENT OF NODE-POSITIVE PATIENTS}

Clinically-positive lymph nodes $(\mathrm{cN}+)$ have usually been defined as pelvic nodes $>8 \mathrm{~mm}$ or abdominal nodes $>10 \mathrm{~mm}$ in maximum short-axis diameter as detected via preoperative computed tomography (CT) or magnetic resonance imaging (MRI). CT/MRI had limited ability in predicting $\mathrm{pN}+$, mainly because of their inability to localize small volume, micro metastatic nodal disease. A multi-institutional study confirmed the poor accuracy of conventional preoperative imaging in assessing nodal disease status: cross-sectional imaging showed sensitivity of $18 \%$ and specificity of $96 \%$ for prediction of lymph node metastases, with accuracy of $78 \%$. Therefore, the pathologic node status was the only reliable predictor of longterm outcome (poor survival), while $\mathrm{cN}+$ status did not show an independent role as a predictor of oncologic outcomes and should be considered carefully before precluding potentially curative treatments (24).

A preoperative imaging method that accurately demonstrates the extent of involvement and therefore may guide the extent of surgical dissection could be desirable for both staging and cure purposes.

Although F-18 FDG PET and PET/CT are now commonly used in imaging of various cancers, their use in BC staging is limited by the high urinary excretion activity in the bladder and ureters. A recent meta-analysis showed a low sensitivity (0.57) and high specificity (0.92) for the detection of metastatic LNs in patients with newly diagnosed BC (25). (Tumour Biol. 2015 May;36(5):3209-14. doi: 10.1007/s13277-014-2361-7. Epub 2015 Mar 26. Diagnostic value of [18F] FDG-PET and PET/CT in urinary bladder cancer: a meta-analysis. Zhang H1, Xing W, Kang Q, Chen C, Wang L, Lu J.)-INSERIRE

Patients with clinically positive LN disease are generally considered for systemic platinum-based $\mathrm{CHT}$ in the induction setting and then RC, as consolidation, in those with a major response to the induction $\mathrm{CHT}(26,27)$.

Neoadjuvant cisplatin-containing combination $\mathrm{CHT}$ for patients with muscle-invasive urothelial carcinoma (UC) has been reported to improve outcomes in several randomized

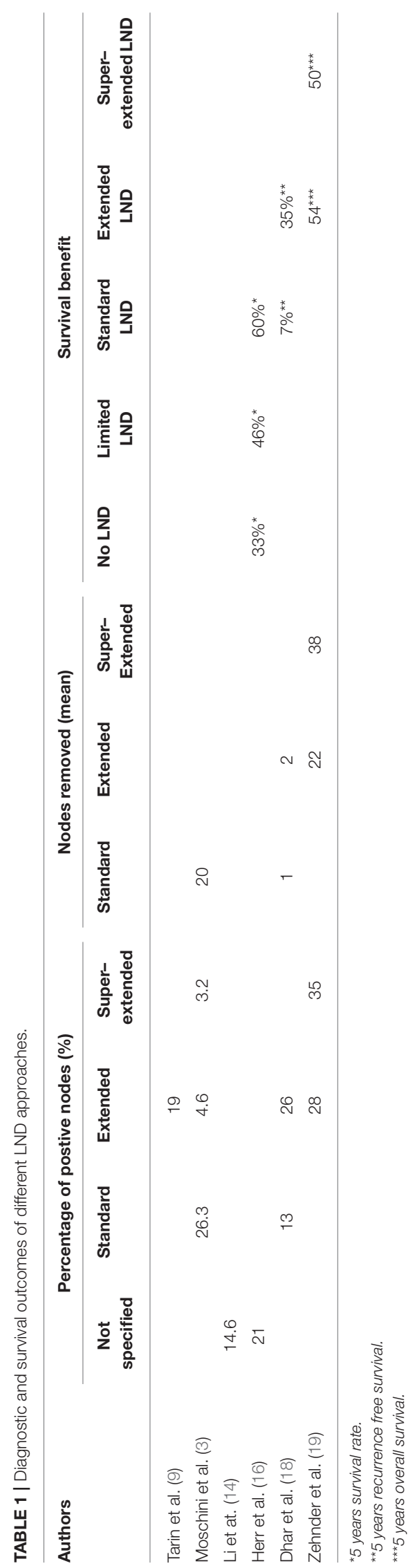


trials (improved overall survival $5-8 \%$ at 5 years). However, most neoadjuvant trials have studied the effect of chemotherapy in patients with clinically negative nodes ( $\mathrm{cN} 0)$ and excluded patients with clinically node positive disease (cN1-3). Clinical LN metastases $(\mathrm{cN}+)$ are common in patients with advanced $\mathrm{UC}$, and the prognosis of patients with $\mathrm{cN}+$ is significantly worse than $\mathrm{cN} 0$. In the absence of visceral metastasis and despite CHT, the reported 5-yr overall (OS) rate was <20\% (28).

LN status after CHT seems to be more important than local tumor status in evaluating survival in $\mathrm{cN}+$ patients, as we can understand from Nieuwenzhuijzen work where a tumor negative bladder combined with tumor negative nodes were associated with improved survival (HR 4.4) as was a tumor negative LN region in the presence of residual bladder disease (HR 2.8) (29).

However, prognosis for $\mathrm{cN}+\mathrm{BC}$ remains poor despite the use of induction $\mathrm{CHT}$ and the use of $\mathrm{CHT}$ only probably represents an under treatment for most patients: an historical series from MSKCC showed that $92 \%$ of patients who did not undergo surgery after major response to CHT died of metastatic disease, while a third of patients who had complete response to $\mathrm{CHT}$ and surgery had long-term survival (30). However, CHT is a milestone in the management of these patients: more recently, Galsky et al. (31) evaluated a large number of patients with $\mathrm{cN}+$ using the National Cancer Data Base and comparing the effects of CHT and/or RC (19). From their results, an multimodal approach integrating perioperative $\mathrm{CHT}$ was associated with better outcomes than $\mathrm{RC}$ alone. The 5-year OS for pre-operative $\mathrm{CHT}$ and $\mathrm{RC}, \mathrm{RC}$ and adjuvant $\mathrm{CHT}$, and RC alone were 31 , 26 , and $19 \%$, respectively. Although the optimal sequence and modalities remain incompletely defined, data suggested a survival benefit with either CHT used before RC or in the adjuvant setting. Overall, the evidence collected suggests a benefit for RC after complete or major response to systemic CHT (32).

In Meijer series 1 of 4 patients showed complete pathologic response to induction CHT with subsequently a significant CSS benefit (median CSS 127 months and 5-year CSS 63.5\%) (33). Several studies assessed pathological and survival outcomes in patients with cN1-3 disease treated with induction CHT and RC. Partial pathological response (pPR) was defined as down staging to non-muscle invasive disease, pT1N0 or less, and complete pathological response (pCR) was defined as pT0N0. In the largest series published, in which 304 patients received

\section{REFERENCES}

1. EAU. Guidelines on Muscle-invasive and Metastatic Bladder Cancer (2018). Available online at: http://uroweb.org/guideline/bladder-cancer-muscleinvasive-and-metastatic/ (Accessed August 22, 2018).

2. Lerner SP, Skinner DG, Lieskovsky G, Boyd SD, Groshen SL, Ziogas A, et al. The rationale for en bloc pelvic lymph node dissection for bladder cancer patients with nodal metastases: long-term results. J Urol. (1993) 149:758-64. Discussion 764-5.

3. Moschini M, Arbelaez E, Cornelius J, Mattei A, Shariat SF, Dell'oglio $\mathrm{P}$, et al. Pattern of node metastases in patients treated with radical cystectomy and extended or superextended pelvic lymph node dissection induction $\mathrm{CHT}$, the $\mathrm{pCR}$ and $\mathrm{pPR}$ rates for the entire cohort were 14.5 and $27 \%$, respectively (34). In the same work, pN0 status, number of LNs removed ( $>15$ ), negative soft tissue surgical margins, and cisplatin-based CHT were independently associated with improvement in overall survival, while no difference was seen in survival outcomes between $\mathrm{cN} 1$ and $\mathrm{cN} 2-3$ patients and between the different chemotherapy regimens. However, a known limitation of this study is that patients who received CHT for $\mathrm{cN}+$ disease but did not proceed to surgery were not included in the analysis, obviously creating a selection bias toward patients with good response to CHT and better prognosis.

High volume nodal disease is very unlikely to be missed by modern imaging techniques, but it is still possible to discover grossly enlarged LN at the time of surgery for RC. This could be in part due to the latency between the initial imaging staging and the date of surgery, with can be significant especially in some systems (35). Even in this context, the available evidence suggests that RC with extensive LND should not be discontinued. As shown by Herr et al in the pre-CHT era (36), a non-negligible proportion of patients (24\%) could be cured with RC and eLND, especially those in whom primary tumor is clinically confined to the bladder (stage T2). On the other hand, results from studies evaluating the outcome when surgery was aborted due to gross $\mathrm{LN}$ involvement and/or extensive extravesical extension revealed poor outcomes (37).

\section{CONCLUSION}

Bilateral pelvic lymphadenectomy is an important part of $\mathrm{RC}$ for BCa. Lymphadenectomy, completed according to the extended template, provides optimal diagnostic and possibly therapeutic results. The final results of two randomized trials (LEA and SWOG S1011) are anxiously awaited to define finally the appropriate extent of PLND. Post chemotherapy surgery may be used in patients with clinically evident pelvic or even retroperitoneal lymph nodal metastases, especially if they have a response to $\mathrm{CHT}$, although definitive evidence is still needed.

\section{AUTHOR CONTRIBUTIONS}

FC, GM, FZ, AM, and FD made a substantial contribution to this article in terms of conceiving, writing, and reviewing the manuscript. doi: 10.1016/j.urolonc.2018.03.002

4. Bruins HM, Skinner EC, Dorin RP, Ahmadi H, Djaladat H, Miranda G, et al. Incidence and location of lymph node metastases in patients undergoing radical cystectomy for clinical non-muscle invasive bladder cancer: results from a prospective lymph node mapping study. Urol Oncol Semin Orig Investig. (2014) 32:24.e13-24.e19. doi: 10.1016/j.urolonc.2012.08.015

5. Stein JP, Lieskovsky G, Cote R, Groshen S, Feng A-C, Boyd S, et al. Radical Cystectomy in the treatment of invasive bladder cancer: long-term results in 1,054 patients. J Clin Oncol. (2001)19:666-75. doi: 10.1200/JCO.2001.19.3.666

6. Abol-Enein H, El-Baz M, Abd El-Hameed MA, Abdel-Latif M, Ghoneim MA. Lymph node involvement in patients with bladder cancer treated with 
radical cystectomy: a patho-anatomical study-a single center experience. $J$ Urol. (2004)172:1818-21. doi: 10.1097/01.ju.0000140457.83695.a7

7. Bochner BH, Cho D, Herr HW, Donat M, Kattan MW, Dalbagni G. Prospectively packaged lymph node dissections with radical cystectomy: evaluation of node count variability and node mapping. J Urol. (2004) 172:1286-90. doi: 10.1097/01.ju.0000137817.56888.d1

8. Leissner J, Ghoneim MA, Abol-Enein H, Thüroff JW, Franzaring L, Fisch M, et al. Extended radical lymphadenectomy in patients with urothelial bladder cancer: results of a prospective multicenter study. J Urol. (2004) 171:139-44. doi: 10.1097/01.ju.0000102302.26806.fb

9. Tarin TV, Power NE, Ehdaie B, Sfakianos JP, Silberstein JL, Savage CJ, et al. Lymph node-positive bladder cancer treated with radical cystectomy and lymphadenectomy: effect of the level of node positivity. Eur Urol. (2012) 61:1025-30. doi: 10.1016/j.eururo.2012.01.049

10. Wallmeroth A, Wagner U, Moch H, Gasser TC, Sauter G, Mihatsch MJ. Patterns of metastasis in muscle-invasive bladder cancer (pT2-4): an autopsy study on 367 patients. Urol Int. (1999) 62:69-75. doi: 10.1159/000030361

11. Bruins HM, Veskimae E, Hernandez V, Imamura M, Neuberger MM, Dahm $\mathrm{P}$, et al. The impact of the extent of lymphadenectomy on oncologic outcomes in patients undergoing radical cystectomy for bladder cancer: a systematic review. Eur Urol. (2014) 66:1065-77. doi: 10.1016/j.eururo.2014.05.031

12. Roth B, Wissmeyer MP, Zehnder P, Birkhäuser FD, Thalmann GN, Krause $\mathrm{TM}$, et al. A new multimodality technique accurately maps the primary lymphatic landing sites of the bladder. Eur Urol. (2010) 57:205-11. doi: 10.1016/j.eururo.2009.10.026

13. Davies JD, Simons CM, Ruhotina N, Barocas DA, Clark PE, Morgan TM. Anatomic basis for lymph node counts as measure of lymph node dissection extent: a cadaveric study. Urology (2013) 81:358-63. doi: 10.1016/j.urology.2012.10.025

14. Li F, Hong X, Hou L, Lin F, Chen P, Pang S, et al. A greater number of dissected lymph nodes is associated with more favorable outcomes in bladder cancer treated by radical cystectomy: a meta-analysis. Oncotarget (2016) 7:61284-94. doi: 10.18632/oncotarget.11343

15. Herr HW, Bochner BH, Dalbagni G, Donat SM, Reuter VE, Bajorin DF. Impact of the number of lymph nodes retrieved on outcome in patients with muscle invasive bladder cancer. J Urol. (2002) 167:1295-8. doi: 10.1016/S0022-5347(05)65284-6

16. Herr HW, Faulkner JR, Grossman HB, Natale RB, deVere White R, Sarosdy MF, et al. Surgical factors influence bladder cancer outcomes: a cooperative group report. J Clin Oncol. (2004) 22:2781-9. doi: 10.1200/JCO.2004.11.024

17. Cole AP, Dalela D, Hanske J, Mullane SA, Choueiri TK, Meyer CP, et al. Temporal trends in receipt of adequate lymphadenectomy in bladder cancer 1988 to 2010. Urol Oncol Semin Orig Investig. (2015) 33:504.e9-504.e17. doi: 10.1016/j.urolonc.2015.07.015

18. Dhar NB, Klein EA, Reuther AM, Thalmann GN, Madersbacher S, Studer UE. Outcome after radical cystectomy with limited or extended pelvic lymph node dissection. J Urol. (2008) 179:873-8. doi: 10.1016/j.juro.2007.10.076

19. Zehnder P, Studer UE, Skinner EC, Dorin RP, Cai J, Roth B, et al. Super extended versus extended pelvic lymph node dissection in patients undergoing radical cystectomy for bladder cancer: a comparative study. J Urol. (2011) 186:1261-8. doi: 10.1016/j.juro.2011.06.004

20. Wright JL, Lin DW, Porter MP. The association between extent of lymphadenectomy and survival among patients with lymph node metastases undergoing radical cystectomy. Cancer (2008) 112:2401-8. doi: $10.1002 / \mathrm{cncr} .23474$

21. Mandel P, Tilki D, Eslick GD. Extent of lymph node dissection and recurrencefree survival after radical cystectomy: a meta-analysis. Urol Oncol Semin Orig Investig. (2014) 32:1184-90. doi: 10.1016/j.urolonc.2014.01.017

22. Gschwend JE, Heck MM, Lehmann J, Ruebben H, Albers P, Heidenreich A, et al. Limited versus extended pelvic lymphadenectomy in patients with bladder cancer undergoing radical cystectomy: survival results from a prospective, randomized trial (LEA AUO AB 25/02). J Clin Oncol. (2016) 34:4503. doi: 10.1200/JCO.2016.34.15_suppl.4503

23. S1011/SWOG n.d. Available online at: https://www.swog.org/clinical-trials/ s1011 (Accessed July 17, 2018).

24. Moschini M, Morlacco A, Briganti A, Hu B, Colombo R, Montorsi F, et al. Clinical lymphadenopathy in urothelial cancer: a transatlantic collaboration on performance of cross-sectional imaging and oncologic outcomes in patients treated with radical cystectomy without neoadjuvant chemotherapy. Eur Urol Focus (2016) 4:245-51. doi: 10.1016/j.euf.2016.11.005

25. Zhang H, Xing W, Kang Q, Chen C, Wang L, Lu J. Diagnostic value of [18F] FDG-PET and PET/CT in urinary bladder cancer: a meta-analysis. Tumour Biol. (2015) 36:3209-14. doi: 10.1007/s13277-014-2361-7

26. Kaag MG, Milowsky MI, Dalbagni G, Thompson RH, Katz D, Reuter VE, et al. Regional lymph node status in patients with bladder cancer found to be pathological stage T0 at radical cystectomy following systemic chemotherapy. BJU Int. (2011) 108:272-7. doi: 10.1111/j.1464-410X.2010. 09981.x

27. von der Maase H, Hansen SW, Roberts JT, Dogliotti L, Oliver T, Moore MJ, et al. Gemcitabine and cisplatin versus methotrexate, vinblastine, doxorubicin, and cisplatin in advanced or metastatic bladder cancer: results of a large, randomized, multinational, multicenter, phase III study. J Clin Oncol. (2000) 18:3068-77. doi: 10.1200/JCO.2000.18.17.3068

28. Urakami S, Yuasa T, Yamamoto S, Sakura M, Tanaka H, Hayashi T, et al. Clinical response to induction chemotherapy predicts improved survival outcome in urothelial carcinoma with clinical lymph nodal metastasis treated by consolidative surgery. Int J Clin Oncol. (2015) 20:1171-8. doi: 10.1007/s10147-015-0839-y

29. Nieuwenhuijzen J, Bex A, Meinhardt W, Kerst J, Schornagel J, Vantinteren $\mathrm{H}$, et al. Neoadjuvant methotrexate, vinblastine, doxorubicin and cisplatin for histologically proven lymph node positive bladder cancer. J Urol. (2005) 174:80-4. doi: 10.1097/01.Ju.0000162018.40891.Ba

30. Herr HW, Donat SM, Bajorin DF. Post-chemotherapy surgery in patients with unresectable or regionally metastatic bladder cancer. J Urol. (2001) 165:811-4. doi: 10.1016/S0022-5347(05)66533-0

31. Galsky MD, Stensland K, Sfakianos JP, Mehrazin R, Diefenbach M, Mohamed $\mathrm{N}$, et al. Comparative effectiveness of treatment strategies for bladder cancer with clinical evidence of regional lymph node involvement. J Clin Oncol. (2016) 34:2627-35. doi: 10.1200/JCO.2016.67.5033

32. Abufaraj M, Dalbagni G, Daneshmand S, Horenblas S, Kamat AM, Kanzaki $\mathrm{R}$, et al. The role of surgery in metastatic bladder cancer: a systematic review. Eur Urol. (2018) 73:543-57. doi: 10.1016/j.eururo.2017.09.030

33. Meijer RP, Mertens LS, Van Rhijn BW, Bex A, Van Der Poel HG, Meinhardt $\mathrm{W}$, et al. Induction chemotherapy followed by surgery in node positive bladder cancer. Urology (2014) 83:134-9. doi: 10.1016/j.urology.2013.08.082.

34. Zargar-Shoshtari K, Zargar H, Lotan Y, Shah JB, van Rhijn BW, Daneshmand S, et al. A multi-institutional analysis of outcomes of patients with clinically node positive urothelial bladder cancer treated with induction chemotherapy and radical cystectomy. J Urol. (2016) 195:53-9. doi: 10.1016/j.juro.2015.07.085

35. Bruins HM, Aben KKH, Arends TJ, van der Heijden AG, Witjes AJ. The effect of the time interval between diagnosis of muscle-invasive bladder cancer and radical cystectomy on staging and survival: a netherlands cancer registry analysis. Urol Oncol Semin Orig Investig. (2016) 34:166.e1-166.e6. doi: 10.1016/j.urolonc.2015.11.006

36. Herr $\mathrm{Hw}$, Donat $\mathrm{Sm}$. Outcome of patients with grossly node positive bladder cancer after pelvic lymph node dissection and radical cystectomy. $J$ Urol. (2001) 165:62-4. doi: 10.1097/00005392-200101000-00015

37. Guzzo TJ, Rogers CG, Deng CY, Bivalacqua TJ, Palapattu GS, Bastian PJ, et al. Outcomes of patients after aborted radical cystectomy for intraoperative findings of metastatic disease. BJU Int. (2008) 102:1539-43. doi: $10.1111 / \mathrm{j} .1464-410 \mathrm{X} .2008 .07877 . \mathrm{x}$

Conflict of Interest Statement: The authors declare that the research was conducted in the absence of any commercial or financial relationships that could be construed as a potential conflict of interest.

Copyright $\odot 2018$ Cattaneo, Motterle, Zattoni, Morlacco and Dal Moro. This is an open-access article distributed under the terms of the Creative Commons Attribution License (CC BY). The use, distribution or reproduction in other forums is permitted, provided the original author(s) and the copyright owner(s) are credited and that the original publication in this journal is cited, in accordance with accepted academic practice. No use, distribution or reproduction is permitted which does not comply with these terms. 